\title{
MAINTENANCE OF GENETIC VARIATION IN APOMICTIC PLANT POPULATIONS
}

\author{
I. SINGLE LOCUS MODELS* \\ D. R. MARSHALL $†$ and 8. S. WEIR $\ddagger \S$ \\ ‡ Department of Statistics, North Carolina State University, P.O. Box 5457 , \\ Raleigh, North Carolina 27650. + CSIRO, Division of Plant Industry, \\ Canberra City, ACT 2601, Australia
}

Received 2.viii.78

\begin{abstract}
Summary
Populations undergoing selfing, outcrossing and apomixis were studied. Genotypic transition equations were established which allow the properties of equilibrium populations to be discussed. Particular attention was paid to the continued maintenance of heterozygosity. It was found that apomixis has no effect on the maintenance of polymorphism in species which otherwise outcross, but in species which partially or completely self, the possibility of the selective maintenance of balanced polymorphism was enhanced.
\end{abstract}

\section{INTRODUGTION}

GAMETOPHYTIC apomixis or agamospermy, asexual reproduction through seed formation, is widely distributed in higher plants. Nygren $(1954,1967)$, for example, listed over 250 species belonging to some 22 families which reproduce wholly or partly by agamospermy. Because of its wide taxonomic distribution, and because of its close relationships with both hybridization and polyploidy, agamospermy has been a continuing, and often controversial, focus of experimental research in plant evolutionary genetics (reviews in Stebbins, 1941, 1950; Gustafsson, 1946, 1947a, b; Battaglia, 1963; Grant, 1971). However, there have been few theoretical studies in this area. Asher (1970), Nace, Richards and Asher (1970), and Templeton and Rothman (1973) recently developed models for the analysis of parthenogenetic animal populations but analogous models for plant populations are still lacking.

The aim of this series of studies is to overcome this deficiency and to develop the appropriate deterministic models to study the effects of facultative apomixis on the genetic structure and evolutionary potential of plant populations. In the first paper we restrict our attention to single locus models.

\section{THE BASIG MODEL}

We consider a single locus in a diploid species with an arbitrary number, say $k$, of alleles. We take the relative frequency of homozygote $A_{i} A_{i}(1 \leqq i \leqq k)$ in generation $n$ to be $f_{i i}^{(n)}$ and that of the heterozygote

* Paper No. 5627 of the Journal Series of the North Carolina Agricultural Experiment Station, Raleigh, North Carolina. This investigation was supported in part by NIH Research Grant No. GM 11546 from the National Institute of General Medical Sciences.

$\S$ To whom reprint requests should be addressed. 
$A_{i^{4}} A_{j}(i \neq j)$ to be $2 f_{i j}^{(n)}$. Further, we take the relative frequency of the $i$ th allele in generation $n$ to be $p_{i}^{(n)}$ with

$$
\sum_{i=1}^{k} p_{i}^{(n)}=1 \text {. }
$$

We assume that in the population under consideration there is a constant probability, $c$, of agamospermy, a constant probability, $s$, of selfing and a constant probability, $t$, of random outcrossing $(c+s+t=1)$. We also assume that all apomictic progeny are genetically identical to their maternal parent; that is, autosegregation (Gustafsson, 1947a) is absent. Finally, we assume the population is large enough for random fluctuations in gene and genotypic frequencies to be unimportant.

We need hardly point out that this model is grossly oversimplified. It is unlikely in practice that the proportions $c, s, t$ are independent of genotype or that they hold uniformly over the whole populations. We might also expect these proportions to change over time. The present model is offered as a first step in the analysis of a complex situation. We believe that even this model has merit in providing a qualitative description of the behaviour of apomictic populations.

Under this model, recurrence equations relating genotpic proportions in successive generations are as follows:

$$
\left.\begin{array}{l}
\left.f_{i i}^{(n+1)}=c f_{i i}^{(n)}+s\left[f_{i i}^{(n)}+\frac{1}{2}\left(\sum_{l \neq i} f_{i l}^{(n)}\right)\right]+t\left(\sum_{l} f_{i l}^{(n)}\right)^{2}\right) \\
f_{i j}^{(n+1)}=c f_{i j}^{(n)}+\frac{1}{2} s f_{i j}^{(n)}+t\left(\sum_{l} f_{i l}^{(n)}\right)\left(\sum_{m} f_{m j}^{(n)}\right), \quad i \neq j
\end{array}\right\}
$$

or alternatively,

where

$$
\left.\begin{array}{l}
f_{i i}^{(n+1)}=\left(c+\frac{s}{2}\right) f_{i i}^{(n)}+p_{i}^{(n)}\left[(s / 2)+t p_{i}^{(n)}\right] \\
f_{i j}^{(n+1)}=\left(c+\frac{s}{2}\right) f_{i j}^{(n)}+t p_{i}^{(n)} p_{j}^{(n)}, \quad i \neq j
\end{array}\right\}
$$

$$
p_{i}^{(n)}=\sum_{l} f_{i i}^{(n)}
$$

It can be easily demonstrated from (1) or (2) that the gene frequencies remain constant over time, as we might expect since there are no directed forces such as mutation or selection acting on the population. However, the genotypic proportions change over generations until an equilibrium is reached. The general $n$-step formulae for the change in genotypic frequencies are as follows:

$$
\left.\begin{array}{l}
f_{i i}^{(n)}=\left(c+\frac{s}{2}\right)^{n} f_{i i}^{(0)}+\left\{1-\left(c+\frac{s}{2}\right)^{n}\right\}\left[(s / 2) p_{i}+t p_{i}^{2}\right] /\left[1-c-\frac{s}{2}\right] \\
f_{i j}^{(n)}=\left(c+\frac{s}{2}\right)^{n} f_{i j}^{(0)}+\left\{1-\left(c+\frac{s}{2}\right)^{n}\right\} t p_{i} p_{j} /\left[1-c-\frac{s}{2}\right]
\end{array}\right\}
$$

where $f_{i i}^{(0)}$ and $f_{i j}^{(0)}$ represent the initial frequencies of genotypes $A_{i} A_{i}$ and $A_{i} A_{j}$, respectively, and 


$$
p_{i}=\sum_{i=1}^{k} f_{i l}^{(n)} \text { for all } n \text {. }
$$

At equilibrium (as $n \rightarrow \infty$ ), we have

$$
\left.\begin{array}{l}
f_{i i}^{(\infty)}=p_{i}^{2}+s p_{i}\left(1-p_{i}\right) /(2-2 c-s) \\
f_{i j}^{(\infty)}=2 t p_{i} p_{j} /(2-2 c-s) .
\end{array}\right\}
$$

The above results can also be expressed in terms of the coefficient of inbreeding, $F$, which is the probability that an individual carries genes identical by descent. For this model, the general $n$-step expression for $F$ is

$$
F^{(n)}=\left(c+\frac{s}{2}\right)^{n} F^{(0)}+s\left\{1-\left(c+\frac{s}{2}\right)^{n}\right\} /(2-2 c-s)
$$

where $F^{(0)}$ is the inbreeding coefficient in some initial reference population. At equilibrium, $(n \rightarrow \infty)$, (5) above becomes

$$
F^{(\infty)}=s /(2-2 c-s)
$$

and we may write (4) as

$$
\left.\begin{array}{l}
f_{i i}^{(\infty)}=p_{i}^{2}+p_{i}\left(1-p_{i}\right) F^{(\infty)} \\
f_{i j}^{(\infty)}=p_{i} p_{j}\left(1-F^{(\infty)}\right) .
\end{array}\right\}
$$

\section{IMPLICATIONS OF THE BASIC MODEL}

An important issue here is the effect of partial apomixis on the levels of heterozygosity maintained in plant populations, given various assumptions about the levels of self- and cross-pollination and the initial composition of the population. Table 1 gives the limiting frequencies of heterozygotes expected at a diallelic locus $(k=2)$ for a range of values of the mating

\begin{tabular}{|c|c|c|c|c|c|c|}
\hline $\begin{array}{c}\text { Level of } \\
\text { agamospermy }(c)\end{array}$ & $\begin{array}{l}\text { Level of } \\
\text { outcrossing }(t)\end{array}$ & $0 \cdot 10$ & 0.20 & 0.30 & 0.40 & 0.50 \\
\hline 0 & $\begin{array}{c}0 \\
0 \cdot 10 \\
0 \cdot 50 \\
0 \cdot 90 \\
1 \cdot 00\end{array}$ & $\begin{array}{c}0 \\
0 \cdot 033 \\
0 \cdot 120 \\
0 \cdot 171 \\
0 \cdot 180\end{array}$ & $\begin{array}{c}0 \\
0 \cdot 058 \\
0 \cdot 213 \\
0 \cdot 302 \\
0 \cdot 320\end{array}$ & $\begin{array}{c}0 \\
0.076 \\
0 \cdot 280 \\
0 \cdot 397 \\
0 \cdot 420\end{array}$ & $\begin{array}{c}0 \\
0.078 \\
0 \cdot 320 \\
0 \cdot 455 \\
0 \cdot 480\end{array}$ & $\begin{array}{c}0 \\
0.09 \\
0.33 \\
0.47 \\
0.50\end{array}$ \\
\hline $0 \cdot 10$ & $\begin{array}{c}0 \\
0 \cdot 10 \\
0 \cdot 50 \\
0 \cdot 90\end{array}$ & $\begin{array}{c}0 \\
0 \cdot 036 \\
0 \cdot 129 \\
0 \cdot 180\end{array}$ & $\begin{array}{c}0 \\
0 \cdot 064 \\
0 \cdot 228 \\
0 \cdot 320\end{array}$ & $\begin{array}{c}0 \\
0 \cdot 084 \\
0 \cdot 300 \\
0 \cdot 420\end{array}$ & $\begin{array}{c}0 \\
0 \cdot 096 \\
0 \cdot 343 \\
0 \cdot 480\end{array}$ & $\begin{array}{c}0 \\
0 \cdot 100 \\
0.35 \\
0.500\end{array}$ \\
\hline 0.50 & $\begin{array}{c}0 \\
0 \cdot 10 \\
0 \cdot 50\end{array}$ & $\begin{array}{c}0 \\
0 \cdot 060 \\
0 \cdot 180\end{array}$ & $\begin{array}{c}0 \\
0 \cdot 107 \\
0 \cdot 320\end{array}$ & $\begin{array}{c}0 \\
0 \cdot 140 \\
0 \cdot 420\end{array}$ & $\begin{array}{c}0 \\
0 \cdot 160 \\
0 \cdot 480\end{array}$ & $\begin{array}{c}0 \\
0.167 \\
0.500\end{array}$ \\
\hline 0.90 & $\begin{array}{c}0 \\
0 \cdot 10\end{array}$ & $\begin{array}{c}0 \\
0 \cdot 180\end{array}$ & $\begin{array}{c}0 \\
0.320\end{array}$ & $\begin{array}{c}0 \\
0 \cdot 420\end{array}$ & $\begin{array}{c}0 \\
0.480\end{array}$ & $\begin{array}{c}0 \\
0.500\end{array}$ \\
\hline
\end{tabular}

\section{TABLE 1}

Expected proportions of heterozygotes in an equilibrium population $(n \rightarrow \infty)$ for various values of $c$ (level of agamospermy), $\mathrm{t}$ (level of outcrossing) and $\mathrm{p}$ (initial gene frequency)

\section{Gene frequency $(p)$}


system parameters, $c$ and $t$, and of $p$, the initial frequency of the less frequent allele.

The values of $f_{12}^{(\infty)}$ in table 1 emphasise three main points. First, in populations which practice mixed agamospermy and random outcrossing $(c+t=1 ; s=0)$, the expected equilibrium levels of heterozygosity are identical to those expected in a fully random-mating population regardless of the value of $c$ (given $c \neq 1$ ). That is, the level of agamospermy does not affect the genotypic composition of an equilibrium population. It does, however, dramatically affect the rate of approach to equilibrium. Fig. 1, which shows changes in the proportion of heterozygotes over time for a population initially composed entirely of heterozygotes $\left(f_{12}^{(0)}=1\right)$ for
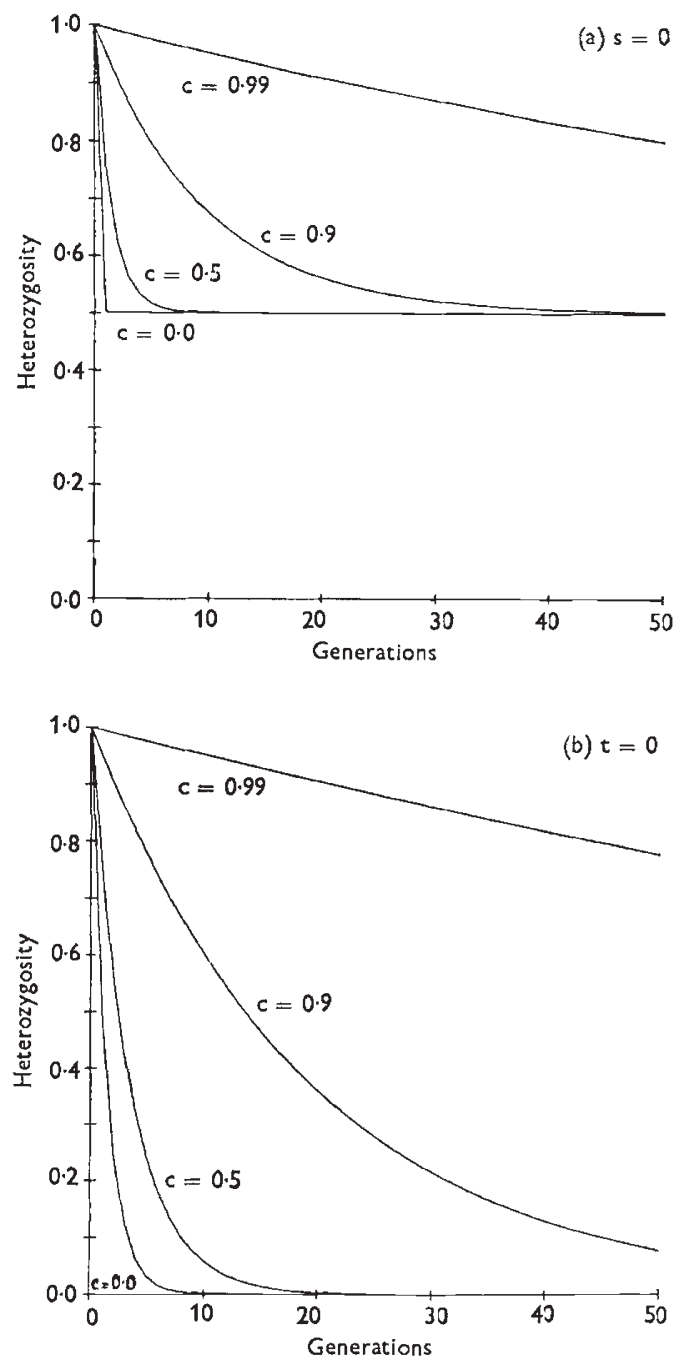

FIG. 1.-Change in heterozygosity with time assuming initial heterozygosity is 100 per cent for (a) species practising mixed outcrossing and agamospermy $(s=0, c+t=1)$ and (b) species practising mixed selfing and agamospermy $(t=0, c+s=1)$. 
various combinations of $c$ and $t$, illustrates this point well. In particular, it emphasises as expected that random-mating populations reach $\mathrm{Hardy}_{-}$ Weinberg equilibrium in one generation, but that this equilibrium is reached more slowly with partial apomixis, and the higher the level of $c$, the slower the approach to equilibrium.

Second, in species which reproduce via mixed agamospermy and selfpollination, the limiting state consists solely of the two homozygotes in the same proportion as the original gene frequencies. No heterozygosity is retained in the equilibrium population. This conclusion holds regardless of the relative magnitudes of $c$ and $s$ provided $t=0$. Here again, however, apomixis does affect the rate of approach to equilibrium.

Third, in species or populations which can reproduce via all three reproductive pathways (apomixis, selfing, or outcrossing), the level of heterozygosity is primarily determined by the relative magnitudes of selfing and outcrossing components. That is, by the ratio $s /(s+t)=s /(1-c)$. For example, the expected heterozygosity in a population with $c=0.90$, $s=0.05, t=0.05$ is identical to that in a population with $c=0, s=0.50$, $t=0.50$. In other words, the imposition of facultative apomixis on a species practising mixed selfing and random mating will not alter the expected genotypic composition of that species provided the relationship between the levels of selfing and outcrossing remains unchanged. As we noted for the other cases, however, apomixis does significantly influence the rate at which the equilibrium population structure is reached.

\section{THE EFFEGTS OF SELEGTION}

We will now consider the effects of differential selective forces on the maintenance of variation in facultative apomicts. Following Workman and Jain (1966) we will consider two models which differ with respect to the time of action of selection and/or the stage of the life cycle at which genotypic frequencies are determined.

\section{(i) Model I}

Under this model it is assumed that the genotypic frequencies are scored just before mating and that all selection has occurred prior to scoring. Again we consider a single locus with an arbitrary number, say $k$, of alleles and we take the relative fitness of the genotype with alleles $A_{i}$ and $A_{j}$ to be $w_{i j}$. Recurrence equations relating genotypic frequencies in successive generations are given in the Appendix, together with an indication of how they may be solved numerically. The numerical treatment follows that of Weir (1970).

\section{(ii) Model II}

This model supposes that genotypic frequencies are scored soon after mating and that no selection occurs between mating and scoring. The genotypic transition equation are also given in the Appendix.

\section{IMPLiGATIONS OF THE SELEGTION MODELS}

In discussing the effects of selection on the maintenance of variation in facultatively apomictic species, we will limit our attention to the simplest 
case of two alleles per locus. Under the assumptions of model I, the equilibrium gene frequencies at a diallelic locus are, from (A.4) and (A.7),

$$
p_{i}=\frac{\left(w_{12}-w_{j j}\right)-\left(w_{12}-w_{i i}\right)\left[s+(2 c+s) F_{12}\right] / 2}{\left(2 w_{12}-w_{11}-w_{22}\right)\left[2-s-(2 c+s) F_{12}\right] / 2}, \quad \begin{array}{ll}
i \neq j \\
i, j=1,2
\end{array}
$$

where, from (A.6),

$$
F_{12}=\frac{2 \bar{w}-(2-s) w_{12}}{2 \bar{w}-(2 c+s) w_{12}} .
$$

A quadratic expression for the mean fitness $\bar{w}$ is found by substituting these expressions into one of the equations of (A.5), e.g.

$$
\begin{aligned}
\bar{w} & =\bar{w}_{1} \\
& =p_{1} w_{11}+p_{2}\left\{w_{12}+\left(w_{11}-w_{12}\right)\left[s+(2 c+s) F_{12}\right] / 2\right\}
\end{aligned}
$$

so that

$$
\begin{aligned}
(1-c-s)\left(w_{11}\right. & \left.+w_{22}-2 w_{12}\right) \bar{w}^{2}+\left\{s(1-c-s)\left[w_{12}\left(w_{11}+w_{22}\right) / 2-w_{11} w_{22}\right]\right. \\
& \left.+(1-s)\left(w_{12}^{2}-w_{11} w_{22}\right)+c(c+s)\left(w_{12}^{2}+w_{11} w_{22}\right)\right\} \bar{w} \\
& +(2 c+s)(1-c-s) w_{12}\left[w_{11} w_{22}-w_{12}\left(w_{11}+w_{22}\right) / 2\right]=0 .
\end{aligned}
$$

Under the assumptions of model II, the expression of equilibrium gene frequencies for $k=2$ are, from (A.9),

$$
p_{i}=\frac{\left(w_{12}-w_{j j}\right)-F_{12}\left(w_{12}-w_{i i}\right)}{\left(2 w_{12}-w_{11}-w_{22}\right)\left(1-F_{12}\right)}, \quad \begin{aligned}
& i \neq j \\
& i, j=1,2
\end{aligned}
$$

where, from (A.10),

$$
F_{12}=\frac{2(c+s) \bar{w}-(2 c+s) w_{12}}{2 \bar{w}-(2 c+s) w_{12}}
$$

and $\bar{w}$ is given by (10) above.

Equilibrium phase diagrams (e.g. Fig. 2) first developed by Hayman and Mather (1953) and used extensively by Workman and Jain (1966) and Asher (1970) offer an effective means of illustrating the types of equilibrium populations possible for various values of mating system parameters, and relative viabilities. The areas marked $A$ and $B$ correspond to populations homozygous for the alternative alleles. Area $C$ represents populations which contain heterozygotes but in a frequency lower than that in a random mating population without selection $(F>0)$. Further, area $D$ represents populations which contain more heterozygotes than expected in a panmictic population in the absence of selection $(F<0)$. Note that we are now using the relative viabilities $w_{11}^{\prime}=w_{11} / w_{12}$ and $w_{22}^{\prime}=w_{22} / w_{12}$, and that attention is restricted to the overdominant cases $\left(w_{11}^{\prime}, w_{22}^{\prime} \leqq 1\right)$. We do not expect to have stable polymorphic populations in cases other than these overdominant ones.

The formulae for the boundary curves can be derived by several methods. For the boundary between regions $C$ and $D$, the "Hardy-Weinberg" curve, we re-write the transition equation (A.l) at equilibrium as 


$$
\left.\begin{array}{l}
\bar{w} f_{11}=w_{11}\left[f_{11}+\frac{s}{2} f_{12}+t\left(f_{12}^{2}-f_{11} f_{22}\right)\right] \\
\bar{w} f_{22}=w_{22}\left[f_{22}+\frac{s}{2} f_{12}+t\left(f_{12}^{2}-f_{11} f_{22}\right)\right] \\
\bar{w} f_{12}=w_{12}\left[\left(1-\frac{s}{2}\right) f_{12}-t\left(f_{12}^{2}-f_{11} f_{22}\right)\right]
\end{array}\right\}
$$

Setting $f_{12}^{2}=f_{11} f_{22}$ in these equations and eliminating $\bar{w}=(2-s) w_{12} / 2$ provides the required boundary

$$
2\left(1-w_{11}^{\prime}\right)\left(1-w_{22}^{\prime}\right)-s\left(1-w_{11}^{\prime} w_{22}^{\prime}\right)=0 .
$$

Note that this result (14) does not involve $c$ and is the same expression derived by Hayman (1953) for mixed selfing and random mating species $(c=0, s+t=1)$. The position of the boundary between $C$ and $D$ is independent of the level of apomixis, and depends only on $s, w_{11}^{\prime}$ and $w_{22}^{\prime}$.

To find the boundary between $A$ and $C$ it is convenient to set $p_{1}=1$ in (8) and $\bar{w}=w_{11}$ in (9) to eliminate $F_{12}$. This procedure yields

$$
2 w_{11}^{\prime}\left(1-w_{11}^{\prime}\right)-2 c w_{22}^{\prime}+2 w_{11}^{\prime} w_{22}^{\prime}(1-t)-s\left(w_{12}^{\prime}+w_{22}^{\prime}\right)=0 .
$$

When $c=0$ this reduces to the result given by Hayman (1953) and if $w_{11}^{\prime}$ and $w_{22}^{\prime}$ are interchanged, it provides the boundary for regions $B$ and $C$. These boundaries do depend on the level of agamospermy then.

Phase diagrams for a range of combinations of mating system parameters are compared in fig. 2. Two points are of note here. First, the imposition of apomixis on a species which practises mixed selfing and random mating so that the ratio of $s / t$ is unaltered, increases both the range of selective values for which the population will be polymorphic and the level of heterozygosity in a polymorphic population (compare figs. $2 \mathrm{D}$ and $2 \mathrm{~B}$ ). Second, for a given level of apomixis, the maintenance of variation is determined primarily by the level of selfing in the population (figs. $2 \mathrm{~A}, \mathrm{~B}, \mathrm{C}$ ).

For species that practise mixed agamospermy and selfing only $(t=0, c+s=1)$, it is necessary to go back to the genotypic transition equations to establish equilibrium frequencies. For model $I$, the great simplification in these equations is seen immediately by setting $t=0$ in equations (13). Equilibrium genotypic frequencies can be found with little difficulty:

$$
\left.\begin{array}{l}
f_{11}=\frac{w_{11} s}{4 D}\left[w_{12}\left(1-\frac{s}{2}\right)-w_{22}\right] \\
f_{22}=\frac{w_{22} s}{4 D}\left[w_{12}\left(1-\frac{s}{2}\right)-w_{11}\right] \\
f_{12}=\frac{1}{2 D}\left[w_{12}\left(1-\frac{s}{2}\right)-w_{11}\right]\left[w_{12}\left(1-\frac{s}{2}\right)-w_{22}\right]
\end{array}\right\}
$$

with

$$
D=w_{12}^{2}\left(1-\frac{s}{2}\right)^{2}-w_{12}\left(w_{11}+w_{22}\right)\left(1-\frac{s}{2}\right)\left(1-\frac{s}{4}\right)+w_{11} w_{22}\left(1-\frac{s}{2}\right)
$$



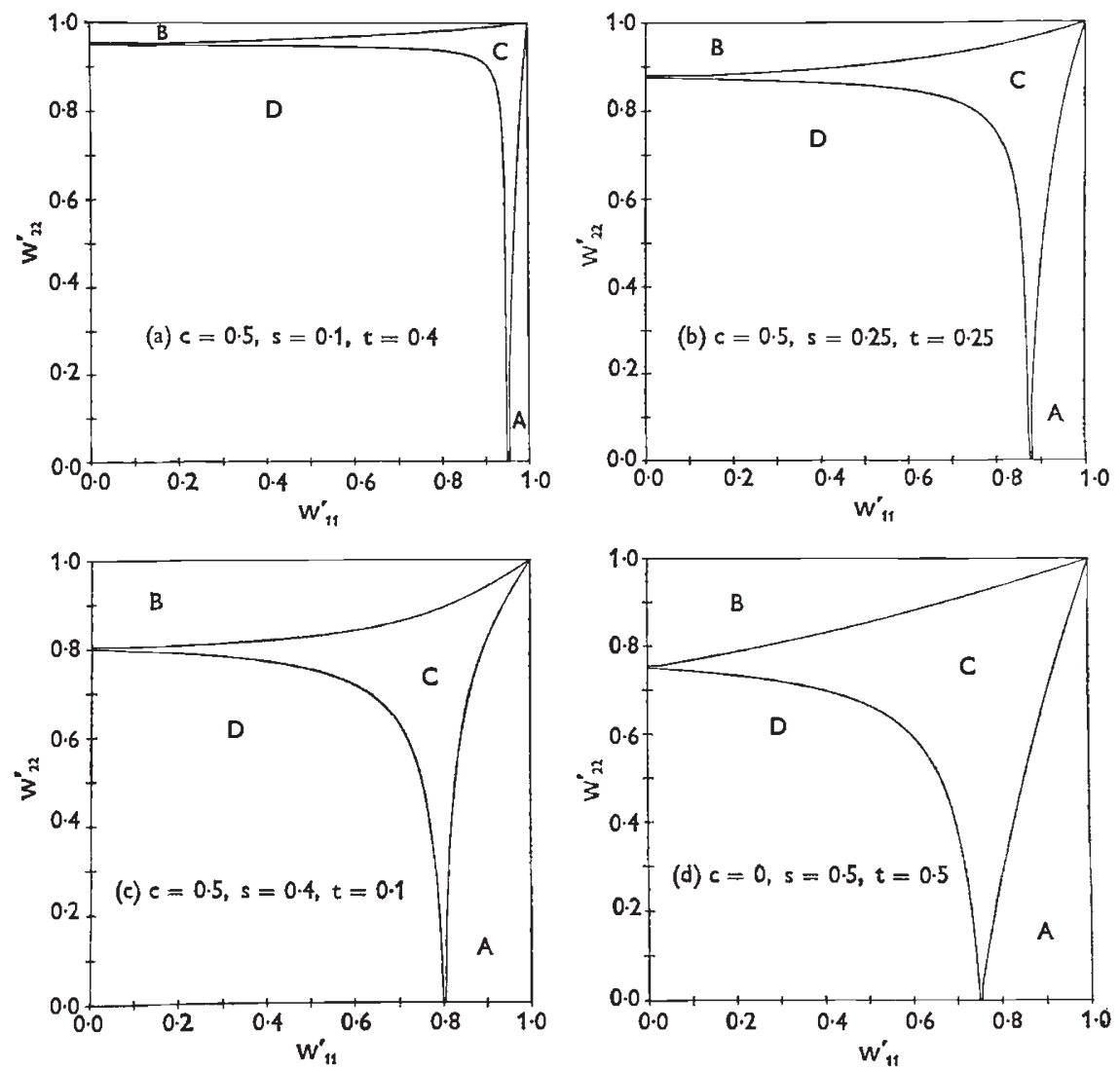

Fig. 2.-Phase diagrams showing range of equilibrium populations for a diallelic locus for $t \neq 0 . w_{11}^{\prime}$ and $w_{22}^{\prime}$ represent the fitnesses of the two homozygotes relative to the heterozygote.

In the phase diagram, the boundary for regions $C$ and $D$ is as before. For regions $A$ and $C$, the boundary is $w_{11}^{\prime}=1-s / 2=(c+s) / 2>\frac{1}{2}$, and for regions $B$ and $C$, it is $w_{22}^{\prime}=(c+s) / 2>\frac{1}{2}$. In the pure selfing case, the corresponding boundaries are $w_{11}^{\prime}=\frac{1}{2}$ and $w_{22}^{\prime}=\frac{1}{2}$ (Hayman, 1953). Evidently the imposition of apomixis on a species that normally reproduces by selfing increases the prospects of maintaining a stable polymorphism at a diallelic locus. Further, the higher the level of apomixis, the less restrictive are the conditions for the maintenance of variation in this class of species. A similar development can be given for model II. Examples of equilibrium phase diagrams for such species are given in fig. 3 .

The other group of species of special interest are those which practise mixed agamospermy and random mating $(s=0, c+t=1)$. The theory leading to equations (8), (9) or (11), (12) is still appropriate and $s=0$ may be substituted directly. Hardy-Weinberg populations are now possible only if $w_{11}^{\prime} w_{22}^{\prime}=1$, which is a result known for random mating. Within the region $w_{11}^{\prime} \leqq 1, w_{22}^{\prime} \leqq 1$, the existence of apomixis has no effect on the maintenance of polymorphism. Heterozygotes will be maintained all over this region (which can thus be labelled $D$ ) for all $c, t$ combinations provided $s=0$. 

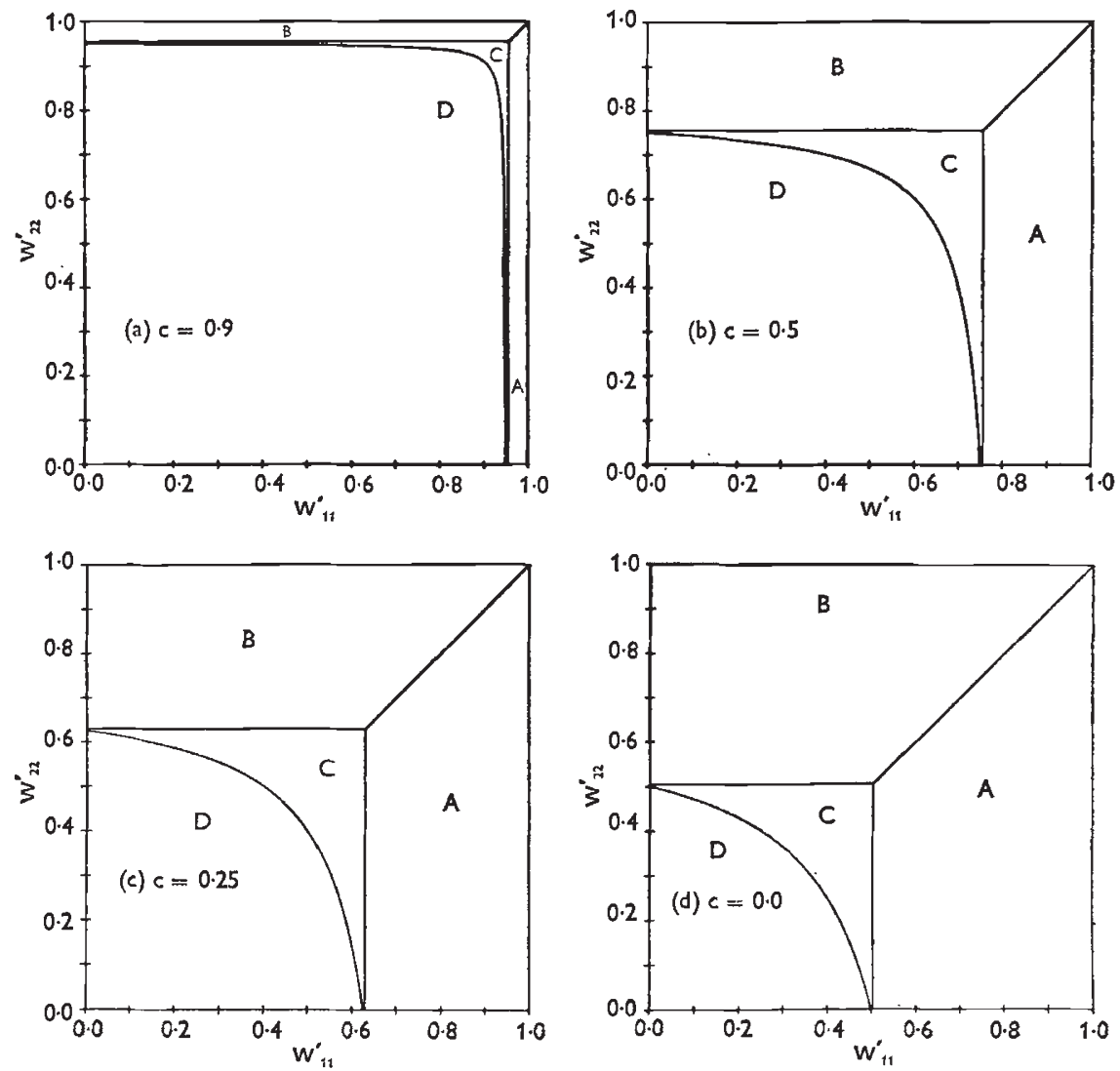

Fig. 3.-Phase diagrams for species which practice mixed selfing and agamospermy $(t=0$, $c+s=1)$. $w_{11}^{\prime}$ and $w_{22}^{\prime}$ represent the fitnesses of the two homozygotes relative to the heterozygote.

\section{Discussion}

The wide taxonomic distribution of agamospermy and the multiplicity of mechanisms underlying asexual seed formation (Gustafsson, 1946; Stebbins, 1950), strongly suggests that this mode of reproduction has arisen independently on many different occasions in higher plants. This suggestion raises two distinct, but obviously interrelated, questions:

(i) Under what circumstances will apomixis become established in a plant species?

(ii) Once apomixis is established in a species, what is its adaptive significance and, in particular, what are its effects on population structure?

The models presented here do not encompass the evolution of apomixis. Consequently, we will not consider the first question further, except to point out that Lloyd (1977) and Maynard Smith (1977) have recently described and discussed simple models of the evolution of mating systems, including agamospermy.

With respect to the second question, strongly contrasting viewpoints have appeared in the literature regarding the evolutionary potential of apomictic 
plant species or species complexes (Grant, 1971). One viewpoint is that agamospecies or agamic complexes have limited evolutionary potential (Darlington, 1939; Stebbins, 1941, 1950; de Wet and Stalker, 1974). From this viewpoint, the principal, or indeed, only, adaptive function of apomixis is that it restores the fertility of individuals that would otherwise be sexually sterile as a consequence of polyploidy and hybridisation. Further, once apomixis becomes established in a species, there is the possibility, if not probability, that it will increase in frequency until the obligate condition is reached. Once this happens the species will lose its capacity for future evolution. This viewpoint was perhaps summarised most succinctly by Darlington (1939) who wrote " apomixis is an escape from sterility, but it is an escape into a blind alley of evolution".

The alternative viewpoint is that apomixis does not reduce, and in favourable circumstances may enhance, the evolutionary potential of plant species (Gustafsson, 1946, 1947a, b; Glausen, 1954; Heslop-Harrison, 1961 ; Rollins, 1967). In this case, emphasis is given to the fact that most species are facultatively apomictic and that sexual and asexual reproduction may coexist indefinitely in an adaptive equilibrium. Thus, from this viewpoint, apomictic species retain their capacity for sexual recombination and hence, progressive evolution. Further, agamospermy facilitates introgressive hybridisation and the exploitation of hybrid vigour, particularly that arising from linked blocks of genes (Clausen, 1961). Finally, gametophytic apomixis facilitates the rapid and indefinite production of highly successful gene combinations, permitting the rapid colonisation and exploitation of available habitats (Clausen, 1954).

The present study confirmed the expectation that obligate apomixis will lead to a loss of variation and evolutionary plasticity. However, it also argued strongly against the viewpoint that apomictic species, if they retain any capacity at all to self or outcross $(c \neq 1)$, necessarily represent " evolutionary dead-ends". It is possible to maintain variation under some circumstances in all populations for $0 \leqq c<1$.

A feature of the present results was the finding that facultative apomixis has no effect on the conditions for the maintenance of heterotically balanced polymorphism in species which otherwise outcross. In contrast, the imposition of apomixis on species practising strict selfing, or mixed selfing and random mating, enhances the possibility for the selective maintenance of balanced polymorphisms. Yet, most facultative apomicts appear to practise mixed agamospermy and random mating. A few practise agamospermy, selfing and random outcrossing, but no species has ever been reported to practise mixed selfing and apomixis (Stebbins, 1950; Grant, 1971).

There are several possible explanations for the fact that apomixis appears to be most prevalent in species where it appears to offer the least advantage in terms of the maintenance of variation in populations. One is that selfing is much more common in facultative apomicts than the previous limited qualitative studies suggested. Another is that higher levels of variation within populations are of little advantage in either the short- or long-term evolution of a species and that, as suggested by Darlington (1939) and de Wet and Stalker (1974), apomixis indeed serves only as an escape from sterility. A third is that the real advantage of apomixis lies in its capacity to ensure the survival of highly fit "coadapted" gene complexes and the maintenance of complex multigene polymorphisms. In this case, we would 
expect apomixis to be at a greater relative advantage in otherwise outbreeding species than those which are completely or partially autogamous because self-pollination itself leads to the maintenance of coadapted complexes. Whatever the explanation, it is clear that there is a substantial need for both quantitative experimental studies and further theoretical studies in this area. The next paper in this series will consider the effects of apomixis on the maintenance of variation at two loci.

\section{REFERENGES}

ASHER, J. H. 1970. Parthenogenesis and gentic variability. II. One locus models for various diploid populations. Genetics, 66, 369-391.

BattagLta, E. 1963. Apomixis. In Recent Advances in the Embryology of Angiosperms, ed. P. Maheshwari, 467 pp. International Society of Plant Morphologists, University of Delhi.

ClaUsEn, J. 1954. Partial apomixis as an equilibrium system in evolution. Caryologia Suppl., 6, 469-479.

CLAUsEN, J. 1961. Introgression facilitated by apomixis in polyploid Poas. Euphytica, 10, 87-94.

Darlington, C. D. 1939. The Evolution of Genetic Systems, 1st Ed., 149 pp. Cambridge Univ. Press, Cambridge.

DE WET, J. M., AND STALKER, H. T. 1974. Gametophytic apomixis and evolution in plants. Taxon, 23, 689-697.

GRANT, v. 1971. Plant Speciation, 435 pp. Columbia University Press, New York.

gustafsson, A. 1946. Apomixis in the higher plants. I. The mechanism of apomixis. Lunds Univ. Arsskr., 43, 1-66.

GUSTAFsson, A. 1947a. Apomixis in higher plants. II. The casual aspect of apomixis. Lunds Univ. Arsskr., 43, 71-178.

GUSTAFSSON, A. 1947b. Apomixis in higher plants. III. Biotype and species formation. Lunds Univ. Arsskr., 44, 183-370.

HAYMAN, B. I. 1953. Mixed selfing and random mating when homozygotes are at a disvantage. Heredity, 7, 185-192.

HAYMAN, B. I., AND MATHER, K. 1953. The progress of inbreeding when homozygotes are at a disadvantage. Heredity, 7, 165-183.

HESLOP-HARRISON, J. 1961. Apomixis, environment and adaptation. In Recent Advances in Botany, Vol. I, pp. 891-895. Proc. 9th Int. Bot. Cong., University of Toronto Press.

LLOYD, D. G. 1977. Genetic and phenotypic models of natural selection. F. Theor. Biol., $69,543-560$.

MAYNARD SMITH, J. 1977. The sex habit in plants and animals. Lecture Notes in Biomathematics, 19, 315-331, eds. F. B. Christiansen and T. M. Fenchel. Springer-Verlag, New York.

NACE, G. W., RIGHARDS, C. M., AND ASHER, J. H. 1970. Parthenogenesis and genetic variability. I. Linkage and inbreeding estimations in the frog, Rana pipiens. Genetics, 66, 349-368.

NYGREN, A. 1954. Apomixis in angiosperms. II. Bot Rev., 20, 577-649.

NYGREN, A. 1967. Apomixis in the angiosperms. Handbook der Pflangenphys., 18, 551-596.

Rollins, R. C. 1967. The evolutionary fate of inbreeders and non-sexuals. Amer. Nat., $101,343-351$.

StebBins, G. L. 1941. Apomixis in the angiosperms. Bot. Rev., 10, 507-542.

STEBbins, G. L. 1950. Variation and Evolution in Plants, 643 pp. Columbia University Press, New York.

TEMPLETON, A. R., AND ROTHMAN, E. D. 1973. The population gentics of parthenogenetic strains of Drosophila mercatorum. I. One locus model and statistics. Theor. Appl. Gen., 43, 204-212.

WEIR, B. s. 1970. Equilibria under inbreeding and selection. Genetics, 65, 371-378.

WORKMAN, P. L., AND JAIN, s. K. 1966. Zygotic selection under mixed random mating and self-fertilization: Theory and problems of estimation. Genetics, 54, 159-171. 


\section{ApPENDix}

\section{(i) Selection model I}

For the general selection model I, we assume the fitnesses $w_{i j}$ to be constant and independent of allelic frequencies. The recurrence equations relating genotypic frequencies in successive generations are as follows:

$$
\left.\begin{array}{l}
f_{i i}^{(n+1)}=\frac{w_{i i}}{\bar{w}^{(n)}}\left\{c f_{i i}^{(n)}+s\left[f_{i i}^{(n)}+\frac{1}{2} \sum_{l \neq i} f_{i l}^{(n)}\right]+t\left(\sum_{m} f_{i m}^{(n)}\right)^{2}\right\} \\
f_{i j}^{(n+1)}=\frac{w_{i j}}{\bar{w}^{(n)}}\left\{c f_{i j}^{(n)}+\frac{s}{2} f_{i j}^{(n)}+t\left(\sum_{i} f_{i l}^{(n)}\right)\left(\sum_{m} f_{m j}^{(n)}\right)\right\}, \quad i \neq j,
\end{array}\right\}
$$

where the mean fitness $\bar{w}^{(n)}$ is such that

$$
\sum_{i} \sum_{j} f_{i j}^{(n+1)}=1
$$

Summing equations (A.1) provides the allelic transition equations

$$
p_{i}^{(n+1)}=\left[\left(c+\frac{s}{2}\right) \sum_{j} w_{i j} f_{i j}^{(n)}+p_{i}^{(n)}\left(\frac{w_{i i} s}{2}+t \sum_{j} w_{i j} p_{j}^{(n)}\right)\right] / \bar{w}^{(n)} .
$$

As has been shown previously (e.g. Weir, 1970) all these transition equations are simplified by the introduction of fixation indices $F_{i j}$ defined as

$$
F_{i j}^{(n)}=1-f_{i j}^{(n)} / p_{i}^{(n)} p_{j}^{(n)}, \quad i \neq j
$$

so that

$$
\begin{aligned}
& f_{i i}^{(n)}=p_{i}^{(n)}\left(p_{i}^{(n)}+\sum_{j \neq i} p_{j}^{(n)} F_{i j}^{(n)}\right) \\
& f_{i j}^{(n)}=p_{i}^{(n)} p_{j}^{(n)}\left(1-F_{i j}^{(n)}\right), \quad i \neq j .
\end{aligned}
$$

In a $k$-allele situation, this means that we have reparameterised $k(k+1) / 2-1$ genotypic frequencies as $(k-1)$ allelic frequencies and $k(k-1) / 2$ fixation indices.

The allelic transition equation (A.2) now becomes

$$
p_{i}^{(n+1)}=p_{i}^{(n)} \bar{w}_{i}^{(n)} / \bar{w}^{(n)}
$$

with mean fitness

$$
\bar{w}^{(n)}=p^{(n)^{\prime}} \Omega^{(n)} p^{(n)}=\sum_{i} p_{i}^{(n)} \bar{w}_{i}^{(n)}
$$

where $p^{(n)^{\prime}}$ is the vector $\left[p_{1}^{(n)}, p_{2}^{(n)}, \ldots, p_{k}^{(n)}\right]$ and $\Omega^{(n)}$ the $k \times k$ matrix with elements

$$
\left.\begin{array}{rl}
\Omega_{i i}^{(n)} & =w_{i i} \\
\Omega_{i j}^{(n)} & =w_{i j}+\left(w_{i i}-w_{i j}\right)\left[s+(2 c+s) F_{i j}^{(n)}\right] / 2 \\
& \neq \Omega_{j i}^{(n)} .
\end{array}\right\}
$$


The marginal mean fitness $\bar{w}_{i}^{(n)}$ is

$$
\bar{w}_{i}^{(n)}=\sum_{j} \Omega_{i j} p_{j}^{(n)} .
$$

Equation (A.3) provides equilibrium conditions. If the absence of $(n)$ or $(n+1)$ superscripts denotes equilibrium values, then we must have the $k$ relations

$$
\bar{w}_{i}=\bar{w} .
$$

A further equilibrium relation is found from the heterozygote transition equations written with fixation indices

$$
\left[1-\frac{(2 c+s) w_{i j}}{2 \bar{w}}\right] p_{i} p_{j}\left(1-F_{i j}\right)=\frac{t w_{i j}}{\bar{w}} p_{i} p_{j}
$$

so that, for non-trivial equilibria $\left(1>p_{i}>0\right)$ we have

$$
F_{i j}=\frac{2 \bar{w}-(2-s) w_{i j}}{2 \bar{w}-(2 c+s) w_{i j}} .
$$

It is necessary that the mean fitness is bounded such that

$$
\bar{w}>(c+s) w_{i i}, \quad \bar{w}>\left(c+\frac{s}{2}\right) w_{i j}, \quad i \neq j,
$$

where the first of these relations follows from the homozygote transition equations.

Evidently $F_{i j}^{(n)}$, and hence $\Omega_{i j}^{(n)}$, are functions of allelic frequencies and change over time. At equilibrium, however, there is no further change and (A.6) allows the matrix $\Omega$ to be expressed solely in terms of the mating system parameters $c, s, t$, the selection coefficients $w_{i j}$, and the mean fitness $\bar{w}$. In other words, the fitness matrix $\Omega$ involves only one unknown, and that is $\bar{w}$.

The equilibrium equations (A.5) are solved by Cramer's rule as

$$
p_{i}=\frac{D_{i}}{\sum_{i} D_{i}}
$$

where $D_{i}$ is the determinant of $\Omega$ with the $i$ th column replaced by l's. It is necessary that the determinant of $\Omega$ is non-zero

$$
|\Omega|=\sum_{i} \bar{w}_{i} D_{i}=\bar{w} \sum_{i} D_{i} \neq 0
$$

and that all $D_{i}$ have the same sign. Equation (A.7) now provides all equilibrium allelic frequencies as functions of $\tilde{w}$, and this mean fitness is found from any one of equations (A.5). In general, these equations must be solved numerically. However, it is possible to obtain explicit expressions for $p_{i}, F_{i j}$ and $\bar{w}$ in a number of special cases, for example, where all heterozygotes have equal fitnesses or where there are only two alleles per locus (Weir, 1970). 


\section{(ii) Selection model II}

The genotypic transition equations are now

$$
\left.\begin{array}{rl}
f_{i i}^{(n+1)}=\frac{c}{\bar{w}^{(n)}} w_{i i} f_{i i}^{(n)} & +\frac{s}{\bar{w}^{(n)}}\left(w_{i i} f_{i i}^{(n)}+\frac{1}{2} \sum_{l \neq i} w_{i l} f_{i l}^{(n)}\right) \\
& +\frac{t}{\left[\bar{w}^{(n)}\right]^{2}}\left(\sum_{l} w_{i l} f_{i I}^{(n)}\right)^{2} \\
f_{i j}^{(n+1)}=\frac{c}{\bar{w}^{(n)}} w_{i j} f_{i j}^{(n)} & +\frac{s}{\bar{w}^{(n)}} \frac{1}{2} w_{i j} f_{i j}^{(n)} \\
& +\frac{t}{\left[\bar{w}^{(n)}\right]^{2}}\left(\sum_{l} w_{i l} f_{i l}^{(n)}\right)\left(\sum_{m} w_{m j} f_{m j}^{(n)}\right), \quad i \neq j .
\end{array}\right\}
$$

As before, the mean fitness $\bar{w}^{(n)}$ ensures that

$$
\sum_{i} \sum_{j} f_{i j}^{(n+1)}=1
$$

and allelic transition equations are found by summing equations (A.8)

$$
p_{i}^{(n+1)}=\sum_{j} w_{i j} f_{i j}^{(n)} / \vec{w}^{(n)}
$$

Once fixation indices are introduced, all the methodology used in model I applies. All equations from (A.3) onwards hold, with the only modifications being in the elements of the fitness matrix

$$
\left.\begin{array}{l}
\Omega_{i i}^{(n)}=w_{i i} \\
\Omega_{i j}^{(n)}=w_{i j}+\left(w_{i i}-w_{i j}\right) F_{i j}^{(n)}, \quad i \neq j
\end{array}\right\}
$$

and in the equilibrium values of the fixation indices

$$
F_{i j}=\frac{2(c+s) \bar{w}-(2 c+s) w_{i j}}{2 \bar{w}-(2 c+s) w_{i j}}, \quad i \neq j .
$$

Now, for a given set of selection coefficients $w_{i j}$ and mating system parameters $c, s, t$, both models I and II have the same equilibrium allelic frequencies and equilibrium mean fitness. Translation from one model to the other is accomplished by the relation between fixation indices

$$
F_{i j_{\mathrm{II}}}=\left[s+(2 c+s) F_{i j_{\mathrm{I}}}\right] / 2
$$

where the subscripts I and II denote the model.

It should be noted that when $c=0$, all of the above theory generalises that of Workman and Jain (1966) and reduces to that of Weir (1970) for species that practise mixed selfing and random mating. 